\title{
POWER SPECTRA OF VELOCITY \\ FLUCTUATIONS IN PLAGES
}

\author{
RICHARD G. TESKE* \\ San Fernando Observatory, The Aerospace Corporation, Los Angeles, Calif., U.S.A.
}

(Received 31 July, 1974)

\begin{abstract}
Spatial power spectral densities of velocities in plages were compared with velocity power spectra of the quiet photosphere. The comparison suggests that photospheric oscillations are not gravity waves.
\end{abstract}

\section{Introduction}

Although extensive theoretical and observational work has been devoted to understanding the physical nature of the solar photospheric oscillations, no unanimity of opinion exists among workers in the field. Because the oscillations are repetitive over intervals of up to an hour or more (Howard et al., 1968; Gonczi and Roddier, 1969; White and Cha, 1973), running modes are not in favor and various trapped modes of propagation have been investigated (Uchida, 1965; Stix, 1970; McKenzie, 1971; Thomas et al., 1971). Although the trapping region is usually assigned to solar atmospheric levels in the lower chromosphere, minority reports by Leibacher and Stein (1971) and by Ulrich (1970) have suggested that the observed oscillations consist of non-propagating evanescent modes excited by pulses from a trapping region below the photosphere. An excellent review of theory has been published by Michalitsanos (1973a).

The trapped modes which may exist in the lower chromosphere can be studied by means of the local diagnostic diagram (cf. Michalitsanos, 1973a, and references cited therein), which is constructed from the dispersion relation and which portrays the domains of temporal frequency and horizontal wavenumber which may be occupied by vertically propagating and non-propagating waves. It is called the 'local' diagnostic diagram because the $(k, \omega)$-loci of boundaries which separate propagating and evanescent modes depend upon the local sound speed, scale height and ratio of specific heats. Additionally, the presence of a magnetic field, which requires introduction of the Alfvén speed into formulation of the dispersion equation, modifies the local diagnostic diagram. Chen and Lykoudis (1972), Michalitsanos (1973b) and Nakagawa et al. (1973) have derived dispersion relations for vertical wave propagation in the presence of a magnetic field and have studied the local diagnostic diagrams for these dispersion equations.

It may therefore be possible to identify the wave mode which is responsible for the photospheric oscillations by examining the oscillations in the magnetic field of an

* Permanent address: University of Michigan, Ann Arbor, Mich. 48104, U.S.A. 
active center and interpreting the observations by means of the diagnostic diagram.

Howard (1967) has shown that the velocity amplitude of oscillations decreases in active centers by some $20 \%$ to $25 \%$ as compared with the quiet photosphere. In an unpublished investigation in $1963 \mathrm{I}$ measured the rms velocity $\left\langle v^{2}\right\rangle^{1 / 2}$ of a number of spectrum lines in and outside of plages near the center of the disk $(0.74 \leqslant \mu \leqslant 0.95)$. The measures were made using isodensitometry of slit spectrograms made with the vacuum spectrograph at the McMath-Hulbert Observatory. From 13 pairs of spectrograms I found that

$$
\frac{\left\langle v^{2}\right\rangle_{\text {plage }}^{1 / 2}}{\left\langle v^{2}\right\rangle_{\text {quiet }}^{1 / 2}}=\frac{0.35 \pm 0.08}{0.40 \pm 0.09},
$$

although the individual pairs of spectra gave ratios varying from 0.74 to 1.3 (two cases gave ratios greater than unity).

In addition to demonstrating a decrease in velocity amplitude, Howard (1967) found that the periods of oscillations were apparently not modified by the magnetic field. His data did not permit examination of possible modification of spatial wavenumber. Nothing is yet known about possible changes in sizes of velocity elements in active centers, nor is it known whether the observed decrease in velocity amplitude is the same for cells of various sizes. An examination of the question affords an opportunity to distinguish between possible wave modes for the oscillations. For example, introduction of a magnetic field into the photosphere and chromosphere will serve to change the local dispersion relation in such a way as to prevent vertical propagation of some oscillations of lower horizontal wavenumber if the oscillations are gravity waves (Michalitsanos, 1973b). The Chen and Lykoudis model (1972) predicts a similar cut-off in plages and also predicts that the diminution in velocity amplitude of gravity waves should be wavenumber dependent.

In an initial approach to the problem I compared power spectral densities of velocity fluctuations in and outside of plages. Single velocity spectroheliograms were obtained and analyzed for this purpose. While these do not isolate the 5-min oscillations, the major component on them is expected to be contributed by the oscillations (Leighton et al., 1962; Deubner, 1969). Although it was hoped that the results might readily distinguish the oscillatory wave mode, this is not yet possible. The results indicate but do not dictate that the oscillations are not gravity waves.

\section{Observations and Their Reduction}

Velocity spectroheliograms and magnetograms for this study were obtained with the $61-\mathrm{cm}$ reflector and spectroheliograph at the San Fernando Observatory (Mayfield, 1971) using the $\mathrm{Ca}$ I $\lambda 6102.7$ line. The entrance slit width corresponded to one arcsec on the solar image; the exit slits, whose width corresponded to $60 \mathrm{~mA}$ in the spectroheliograph focal plane, were centered at $60 \mathrm{~mA}$ from the line core. For the magnetograms, the violet wing of the line was used.

Two velocity spectroheliograms of excellent quality were selected for analysis, one 
from 10 August 1973 (McMath Plage No. 12476) and one from 28 August 1973 (McMath Plage No. 12501). On the 10 August velocity picture, features of size $\sim 1^{\prime \prime}$ are clearly visible. The 28 August velocity picture is only slightly inferior to this.

Individual scenes from both output channels were scanned and digitized using an Optronics P-1000 high-speed film reader with a 25 micrometer scanning aperture which was equivalent to $0.42^{\prime \prime}$ on the $\operatorname{Sun}\left(\lambda_{\text {Nyquist }}=612 \mathrm{~km}\right)$. Data points were also separated

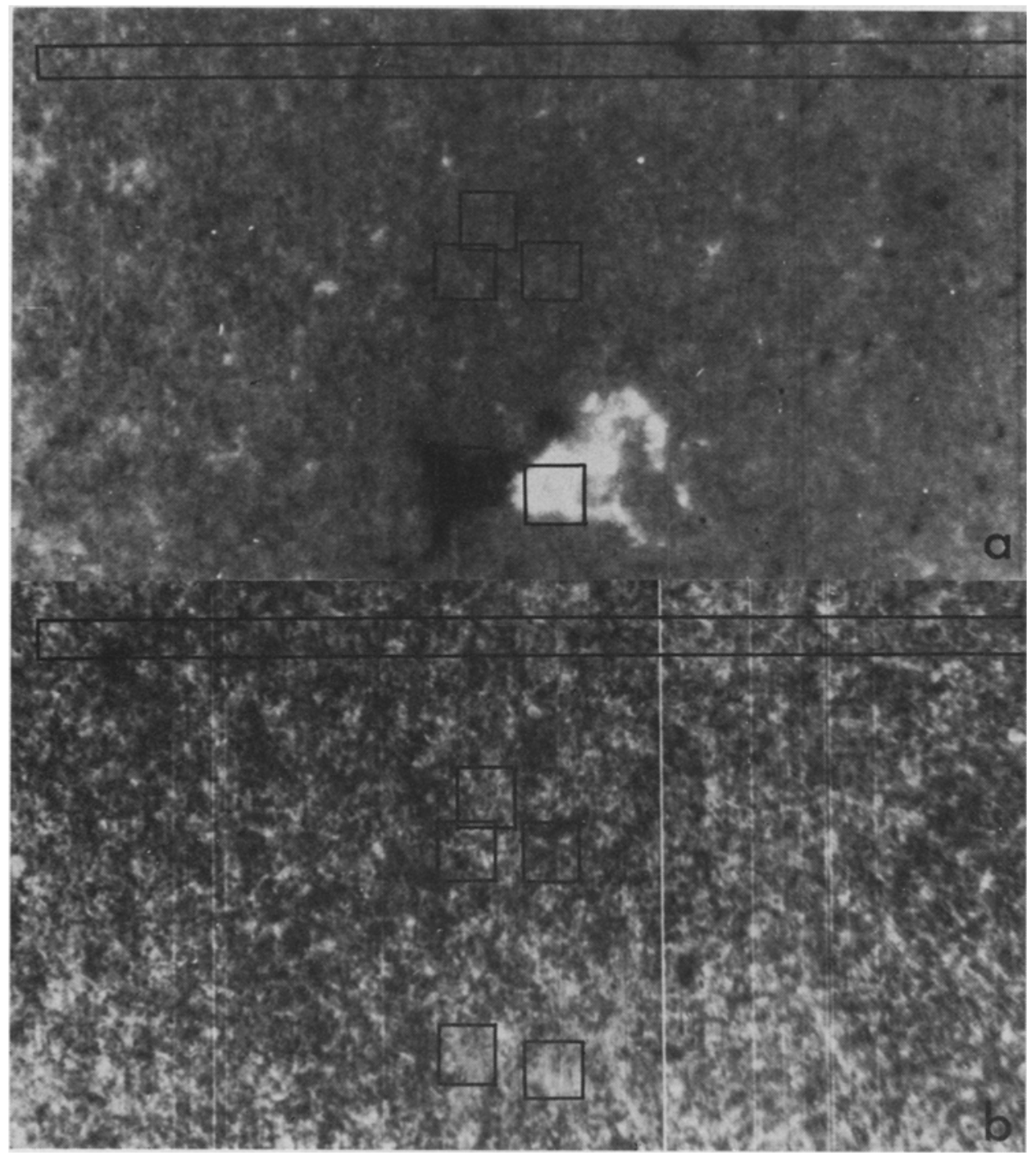

Fig. 1. (a) A portion of the doubly-cancelled magnetogram for 10 August showing one of the plage regions (McMath No. 12476) that was investigated. Dark signifies field directed away from observer. North is upward and east to the left. (b) A portion of the Dopplergram for 10 August. Dark signifies motion away from the observer. The squares outline small $27 \times 27^{\prime \prime}$ regions that were autocorrelated. The rectangular box outlines the region selected for derivation of forty quiet photosphere power spectra. North is upward and east to the left. 
by $0.42^{\prime \prime}$. The individual scenes were subtracted in a CDC $7600 / 6400$ computer to produce discrete digitized versions of the velocity field. In addition, darkroom subtractions (Leighton et al., 1962) of both velocity and magnetic data were carried out to provide guides for subsequent computer analysis of the digital data. The darkroomprocessed version of one of the velocity pictures is shown in Figure 1 together with the accompanying doubly-cancelled magnetogram.

The complete calibration procedure for the digitized data is described by Teske (1974b).

\section{Results}

\subsection{RMS VELOCITY, BRIGHTNESS AND BRIGHTNESS-VELOCITY CORRELATION}

Determination of the Doppler signal, brightness signal and brightness-velocity correlation coefficient is described by Teske (1974b): the procedure is that devised by Leighton et al. (1962). Five small $27 \times 27^{\prime \prime}$ regions in each of the two velocity pictures were analyzed to obtain the rms velocity fluctuation $\left\langle v^{2}\right\rangle^{1 / 2}$, the rms brightness fluctuation $\left\langle\beta^{2}\right\rangle^{1 / 2}$ and the brightness-velocity correlation coefficient $C_{\beta v}$. In each velocity picture two of these regions were selected to be in an active center (see Figure 1) and three were in the quiet photosphere. These quiet comparison areas were selected to lie at very nearly the same distance from disk center as were the magnetic areas.

Table I compares the means of brightness and velocity fluctuations that were derived

TABLE I

\begin{tabular}{lllllll}
\hline Date & $\mu$ & $\left.\left\langle v^{2}\right\rangle\right\rangle^{1 / 2}$ & $\left\langle\beta^{2}\right\rangle^{1 / 2}$ & $C_{\beta v}$ & AC FWHM $(\mathrm{km})$ & Corrected $\left\langle v^{2}\right\rangle^{1 / 2}$ \\
\hline 10 Aug 1973 & & & & & & \\
Plage & 0.87 & $0.35(0.40)$ & 0.052 & -0.34 & $2550 \pm 50$ & $0.36(0.41)$ \\
Quiet & 0.88 & 0.38 & 0.036 & +0.02 & $2300 \pm 140$ & 0.39 \\
28 Aug 1973 & & & & & & \\
Plage & 0.56 & $0.30(0.34)$ & 0.032 & -0.21 & $1550 \pm 50$ & $0.34(0.38)$ \\
Quiet & 0.57 & 0.43 & 0.032 & -0.02 & $2700 \pm 170$ & 0.44 \\
\hline
\end{tabular}

separately for quiet and active regions. Although essentially zero in the quiet regions, the brightness-velocity correlation coefficient is decidedly negative in the active centers (Table I). There is thus a strong tendency for red shifts to associate with weakened line wings: it is likely that local downdrafts at the positions of magnetic fields (Frazier, 1971; Giovanelli and Ramsay, 1971; Stenflo, 1973) have caused $C_{\beta v}$ to be negative in plages, although these downdrafts are probably not resolved in the San Fernando spectroheliograms.

rms velocities in Table I were obtained from calibrations which use the line profile on the quiet disk. For this reason, the rms velocity for the active centers is a lower limit because the line profile becomes less steep in magnetic areas of the disk (Sheeley and Engvold, 1970). These authors suggested a correction factor for the change in line slope of no more than 1.15 , to be applied to velocities based on the quiet disk line 
profile. Use of this factor in the present data yields the values shown in parentheses in column 3 of Table I. We thus derive for the 10 August data $(\mu=0.87)$ a plage rms velocity which is approximately the same as the photospheric value measured at the same distance from disk center while for the 28 August data $(\mu=0.56)$ the plage velocities are roughly $20 \%$ below the local quiet photospheric value.

One possible explanation is that bulk gas motions in magnetic regions are constrained to be almost entirely vertical: in the quiet photosphere we observe a combination of vertical and horizontal components of velocity while in the plage only the vertical component is seen. However, in the unpublished work cited above the range in the ratio $\left\langle v^{2}\right\rangle_{\text {plage }}^{1 / 2} /\left\langle v^{2}\right\rangle_{\text {quiet }}^{1 / 2}$ near disk center includes the values obtained here. Moreover, Howard's (1967) observations also yield a genuine decrease in $\left\langle v^{2}\right\rangle_{\text {plage }}^{1 / 2}$ near disk center, indicating that the vertical component of velocity is affected by a magnetic field. Whether bulk gas motions are or are not constrained to vertical directions is not expected to be an important consideration in the power spectrum comparisons to be made later, since it is the horizontal wavenumber which is of interest.

A second possible explanation for the differences in the velocity comparison between plage and quiet regions has to do with the effects of seeing on the measured $\left\langle v^{2}\right\rangle^{1 / 2}$. For example, if velocity cells are smaller in plages than in quiet regions, seeing effects which tend to decrease the measured rms velocities will cause a greater decrease in the plage value than in the quiet photosphere value. It may be shown that (Mattig et al., 1969), for a gaussian seeing function of dispersion $b$, the true $\left\langle v^{2}\right\rangle^{1 / 2}$ for cells of size $L$ is suppressed by

$$
\left\langle v^{2}\right\rangle_{\text {meas }}^{1 / 2}=\left\langle v^{2}\right\rangle_{\text {true }}^{1 / 2}\left[\exp \left(-\frac{b^{2} \pi^{2}}{4 L^{2}}\right)\right] .
$$

We may evaluate the effects of seeing by determining a mean cell size and a seeing parameter for the observations. An estimate of the mean cell size can be obtained from velocity autocorrelation functions and the seeing parameter estimated from velocity power spectral densities.

Two dimensional autocorrelation (AC) functions of velocity were computed for each of the $27 \times 27^{\prime \prime}$ squares. The AC functions for the quiet areas were discussed in a previous paper (Teske, 1974a) where it was pointed out that the full width at half maximum (FWHM) of the AC functions along the direction of the spectroheliograph entrance slit is a good guide to a mean size for velocity cells. The sixth column of Table I shows the mean FWHM for the AC functions; we take these to give $L$ in Equation (1). The data show that velocity elements in the plage on 28 August were significantly smaller than on the quiet photosphere, and will thus have been more moditied by seeing.

One dimensional power spectral densities, which will be discussed in the next section, show a white noise 'toe' starting at wavenumbers $\log k=-2.2$ (10 August and at $\log k=-2.3$ (28 August). We take the wavenumber at which the white noise begins as indicating the combined effects of seeing, telescope aperture and digitization noise and derive from that an effective seeing parameter $b$. For example, $\log k=-2.2$ on 10 
August corresponds to a cell size of $\pi / 0.0063=500 \mathrm{~km}$ and thus to a seeing disk of dispersion $b=250 \mathrm{~km}$. Similarily we find for 28 August that $\log k=-2.3$ corresponds to $b=315 \mathrm{~km}$.

With these values of $b$ and the mean cell sizes $L$, we obtain via Equation (1) the corrected $\left\langle v^{2}\right\rangle^{1 / 2}$ given in the last column of Table I. It is apparent that the corrections have not removed the difference in $\left\langle v^{2}\right\rangle^{1 / 2}$ between plage and quiet disk for the 28 August data. The difference is therefore independent of smoothing effects and likely intrinsically solar.

\subsection{ONE DIMENSIONAL POWER SPECTRAL DENSITIES}

Velocity power spectral densities both in plages and in the quiet photosphere were calculated using standard mean lagged product methods (Blackman and Tukey, 1958; Reiling, 1971; Beckers and Parnell, 1969). These one dimensional power spectra were obtained from data strings on the velocity pictures taken parallel to the entrance slit to avoid as much as possible the broadening effects of the slit. In carrying out the intended comparison it would usually be desirable (e.g., Canfield and Mehltretter, 1973 ) to correct the power spectra for seeing, for telescope aperture and for photographic grain and digitization noise. However, we intend to compare directly the power spectra of quiet and active regions which obtained from the same spectroheliograms under identical conditions. Because the corrections to be applied would therefore be identical for both spectral densities, the intended comparison can be done without using these corrections.

To achieve an acceptable resolution at low spatial frequencies the use of long data segments on the velocity pictures is required. Power spectra for the quiet photosphere were obtained from 960 data points $(294000 \mathrm{~km})$ lagged 300 times $(91800 \mathrm{~km})$. The plages were too small to permit use of single long data segments. Instead, pairs of 170-pixel data strings $(52000 \mathrm{~km})$ were joined together and lagged 200 times $(61000 \mathrm{~km})$. As a result the plage power spectra contain some spurious power due to the inevitable phase shift at the point where data strings join (White and Cha, 1973). They are also somewhat unstable because of sampling problems connected with the excessive lag. Averaging of individual, raw power spectra helps to some extent to decrease the contributions of both effects but will not remove them entirely. Figure 2 shows some averaged raw power spectra for quiet and active regions that were obtained. These have not otherwise been smoothed.

They are essentially featureless over the range $-3.4 \lesssim \log k \leq-2.3$. Peaks which do appear in that range are probably not reliable. We note the position of the onset of the noise 'toe' at high wavenumbers, a flat white noise region that is most evident in the power spectra for the quiet photosphere. The wavenumber at which this noise begins was used in the last section to estimate the seeing parameter $b$.

Such power spectra should be viewed with care: there is a question as to what information they truly convey about the spatial organization of velocity elements in the solar atmosphere. Previously (Teske, 1974a) it was concluded that one dimensional power spectral densities satisfactorily represent the available information when a 
statistically representative data sample is used, as is the case here. It was also concluded that spatial aperiodicities in the organization of the oscillatory cells prevented their clear portrayal in power spectra; the cells are smeared into a broadened wavenumber band by their quasi-random distribution. Recent numerical experiments by Lynch (1974) in connection with his studies of oscillations have elaborated upon this problem. We thus expect that cells of a given size are to be found distributed across a band of wavenumbers in our power spectra. Additionally, some redistribution of power has occurred in the plage spectra because of the sampling technique that was used.

With these problems in mind we turn to the comparison of averaged unsmoothed power spectral densities for quiet and active regions which is shown in Figure 3. In the figure we have plotted $k P=$ (wavenumber) $\times$ (power per unit wavenumber) as a function of $\log k$ for quiet regions $\left(k P_{Q}\right)$ and for the power difference between quiet and active regions $\left(k P_{Q}-k P_{P}\right)$. These mean power spectra were obtained from 40 spectra in quiet regions and 20 in plages, in each of the velocity pictures. A power deficiency in plages appears as a positive value of $\left(k P_{Q}-k P_{P}\right)$ and a power excess would be signalled by a negative value. Two $\left(k P_{Q}-k P_{P}\right)$-curves are shown: one for velocities obtained from a direct calibration in terms of the photospheric line profile

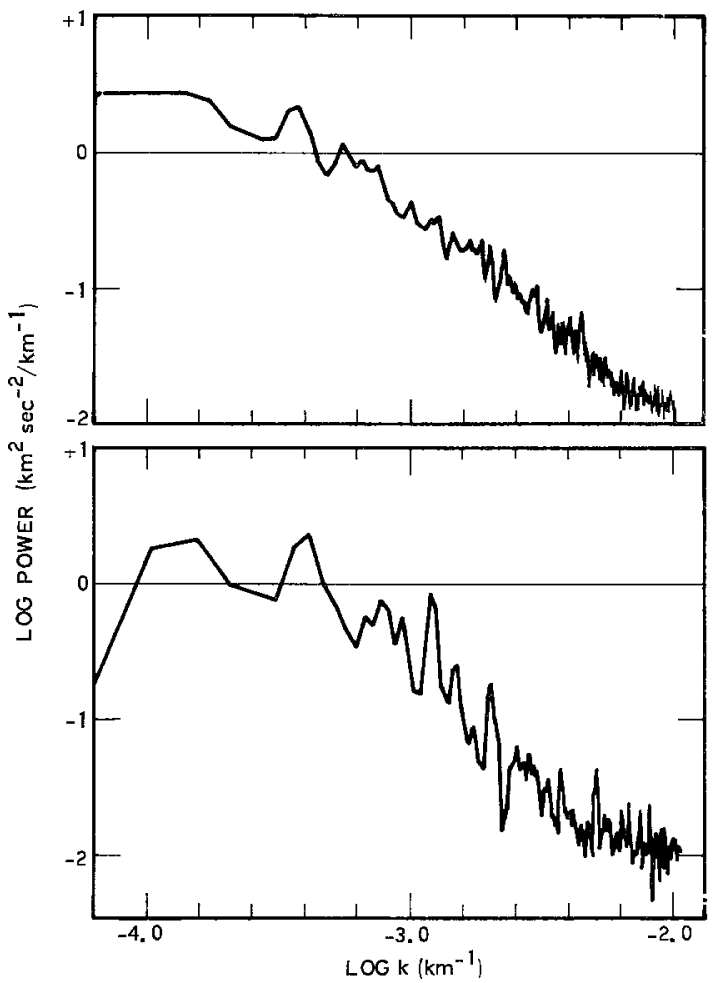

Fig. 2.a. Raw power spectra for 10 August. Upper: average of 20 power spectra for quier disk. Lower: average of 10 power spectra for plage. 


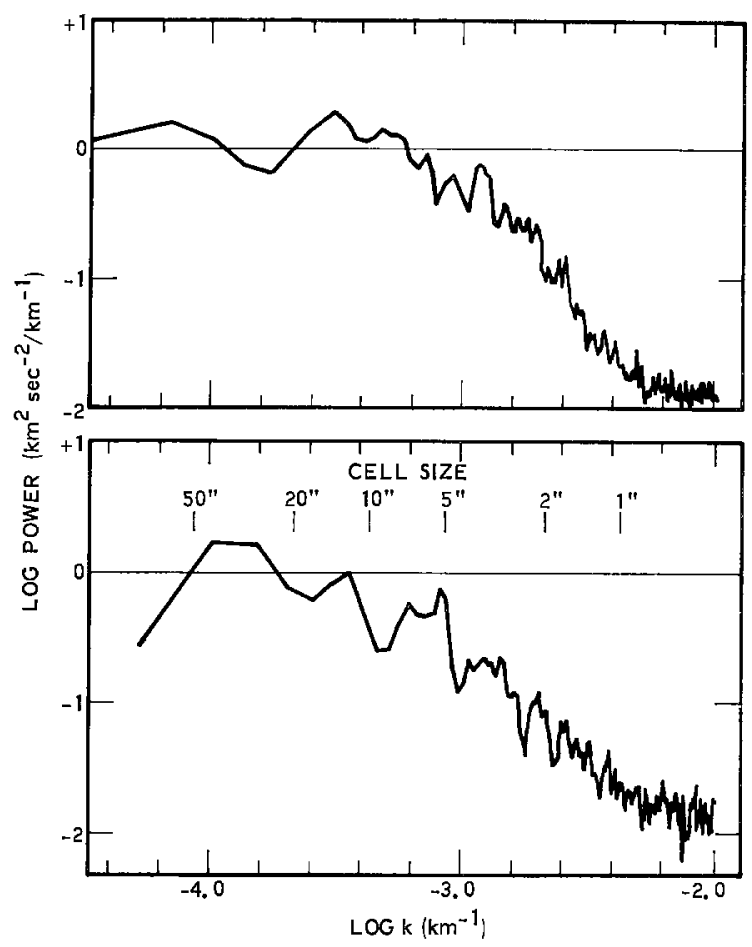

Fig. 2b. Raw power spectra for 28 August. Upper: average of 20 power spectra for quiet disk. Lower: average of 10 power spectra for plage.

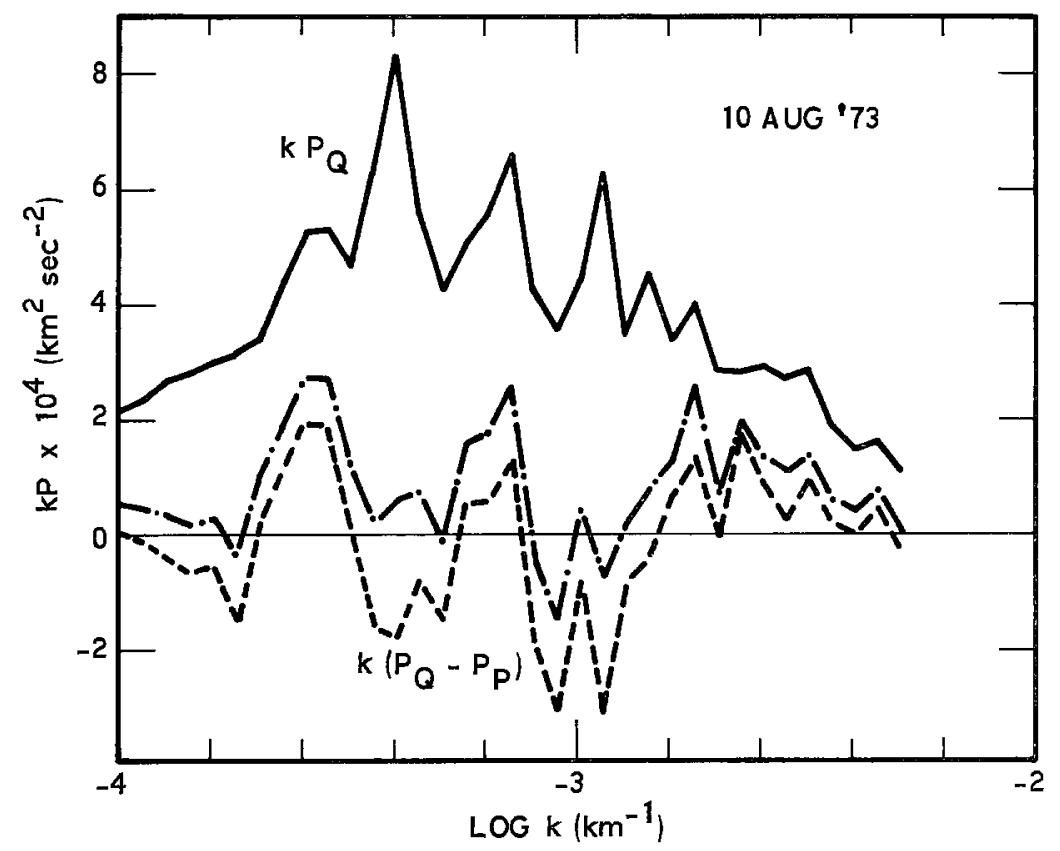

Fig. 3a. Power spectra for 10 August. Solid line: average of 40 power spectra of quiet disk. Dashed line: difference of averaged quiet and plage power spectra for corrected plage velocities. Dot-dash line: difference of power spectra for uncorrected plage velocities. 


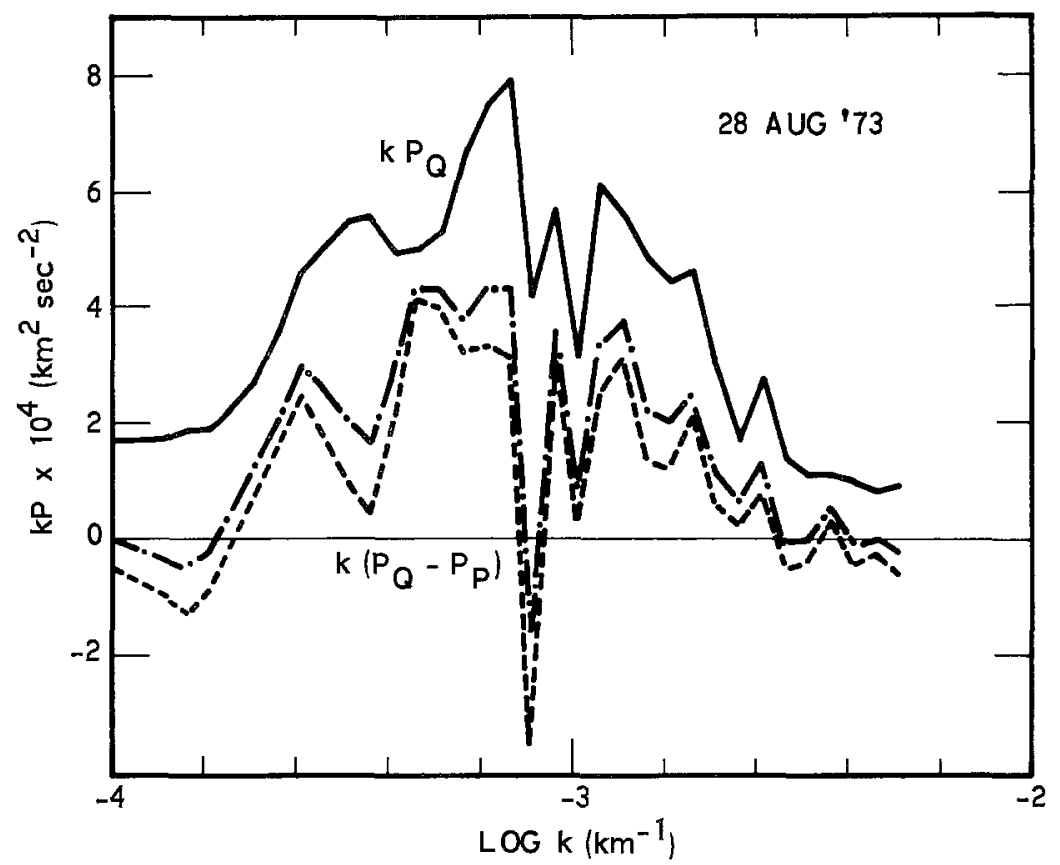

Fig. 3b. Power spectra for 28 August. Solid line: average of 40 power spectra for quiet disk. Dashed line: difference of averaged quiet and plage power spectra for corrected plage velocities. Dot-dash line: difference of power spectra for uncorrected plage velocities.

and one which takes into account Sheeley and Engvold's (1970) suggested maximum correction factor for plage velocities of 1.15. These two curves then let us see the likely range of comparison between power spectra in quiet and active regions.

As a check upon the relative powers we may compute the ratios $\left\langle v^{2}\right\rangle_{\text {plage }}^{1 / 2^{\top}} /\left\langle v^{2}\right\rangle_{\text {quiet }}^{1 / 2}$ by integration of the areas between the curves in Figure 3 and compare them to the same ratios derived from Table I. For the uncorrected plage velocities we get for 10 August: 0.92 from Table I and 0.88 from Figure 3; for 28 August we get: 0.70 from Table I and 0.72 from Figure 3. Thus essentially all the power contributing to the calculated rms velocities is depicted in the power spectra.

The $95 \%$ confidence interval for the curves in Figure 3, as computed from the variance of the mean, spans a total of 4.2 units in $k P$ at $\log k=-3.56$, and spans a total of 3.4 units in $k P$ at $\log k=-2.2$.

We are therefore reluctant to claim that any of the peaks and dips in Figure 3 are real, especially the suggestion of a plage power excess near $\log k=-3$. Rather it appears that the deficiency in plage power on 28 August extends at least across the wavenumber range $-3.7 \$ \log k \lesssim-2.6$ in a roughly uniform way for both the corrected and uncorrected data. For the 10 August data the matter is unclear. At least in the corrected plage power it may be that a deficiency exists only in the vicinity of $\log k \sim$ -2.5 to -2.8 , if anywhere at all. We would expect that the wavenumber smearing of 
oscillatory power in the 10 August plage might extend into these higher wavenumbers, yet an indication of a weak power deficiency seems to persist there. The power spectra for quiet regions suggest a broad distribution peaking near $\log k \sim-3.0$ to -3.2 , at cell sizes in the range 3000 to $5000 \mathrm{~km}$. The breadth of the peak to some unknown extent represents blurring effects introduced by random spacing of the oscillatory elements (Teske, 1974a).

The possible relationship of these power spectra to some models of the oscillations is discussed in Section 4.

\section{Discussion}

Theoretical arguments have been offered favoring gravity waves as the mode for photospheric oscillations. The gravity wave model of Thomas et al. (1971) was extended to include magnetic fields by Chen and Lykoudis (1972). Their model predicted an eigenmode with a $258 \mathrm{~s}$ period for elements of $1500 \mathrm{~km}$ wavelength $(\log k=-2.38)$. It also predicted a decrease of $\left\langle v^{2}\right\rangle^{1 / 2}$ by $25 \%$ in plages, although the model may in fact be adjusted to reflect practically any observed power deficiency at short spatial wavelengths. So far as we may trust the present power spectra, they show that only a small diminution of velocity power occurs at the shortest wavelengths, if any.

Calculations for a range of wavenumber which we carried out with their model for $H=100 \mathrm{G}$ and $H=200 \mathrm{G}$ and a period of $300 \mathrm{~s}$ showed that the ratio $\left\langle v^{2}\right\rangle_{\text {plage }}^{1 / 2} /$ $\left\langle v^{2}\right\rangle_{\text {quict }}^{1 / 2}$ increases as $\log k$ decreases. That is, $\left(k P_{Q}-k P_{P}\right)$ in Figure 3 should decrease from some positive value towards zero in going from $\log k=-2.2$ to $\log k=-2.55$. As respects the gross trend of $\left(k P_{Q}-k P_{P}\right)$ in our data at high wavenumbers, the prediction is not verified.

On the other hand Michalitsanos's (1973b) model for an inclined magnetic field shows that the wavenumber cut-off of the gravity wave mode retreats to higher wavenumbers in the presence of a field, as does the Chen-Lykoudis model. In consequence the $\left(k P_{Q}-k P_{P}\right)$-curve should remain near zero at the highest $k$ and then rise to positive values at a spatial cut-off wavenumber dictated by the field strength and inclination. The gross trend of the material in Figure 3 may not be in disagreement with this prediction. While the apparent cut-off at $\log k \sim-2.6$ suggested by the 28 August data is much smaller than Michalitsanos's model predicts, wavenumber smearing in our power spectra may have introduced some confusion.

Depending upon the height distribution and direction of the magnetic field in the two plages investigated, these results may not therefore rule out the existence of a gravity wave mode at the shortest wavelengths. But the considerable amounts of power at lower wavenumbers both in quiet and in magnetic regions suggests that, if present, this mode does not dominate the oscillations.

If we overlook the apparent but probably spurious plage power excess near $\log k=$ $=-3$, the major power suppression in the plage on 28 August occurs in the midfrequency range, peaking near the maximum of the $k P_{Q^{-}}$-curve, which in turn probably represents the oscillatory cells of size 3000 to $5000 \mathrm{~km}$. Although the plage power decrease appears roughly uniform with wavenumber, smoothing of the power spectra 
suggests that the decrease in plage power reaches a maximum of around $40 \%$ near cell sizes 3000 to $5000 \mathrm{~km}$ and declines to a deficiency of around $15 \%$ at cell sizes near $12000 \mathrm{~km}$ and $1400 \mathrm{~km}$. The 10 August data, on the other hand, indicate that plage power is suppressed if at all at only the shortest wavelengths. In each case there does seem to be a differential damping of velocity fluctuations in a magnetic field which depends upon sizes of velocity elements, although the results are not consistent with one another. Whether the source of this inconsistency should be sought in the magnetic field strengths of the two regions - the 28 August plage had considerably stronger fields than the 10 August plage did - or in some other characteristic of the regions, cannot be said right now.

More data should be analyzed in an attempte to understand whether the apparent plage power excess near $\log k=-3$ is or is not real. While we prefer to discount it in the present data, we note that it appears in both sets of power spectra.

\section{Summary}

Velocity spectroheliograms were used to study velocity and brightness fluctuations in and outside of active centers on the Sun. The brightness-velocity correlation in plages is negative, suggesting again the presence of downdrafts in the plages. After cor rection for line shape and for seeing, the rms velocity in one plage was about $15 \%$ below the value for the quiet photosphere while in a second plage no net deficiency was found. Mean sizes for velocity cells in the former plage (28 August) were smaller than in the quiet photosphere and were comparable to quiet regions in the latter plage (10 August). A comparison of power spectral densities of velocity fluctuations indicated that in the 28 August plage velocities were damped differentially in the approximate wavenumber range $-3.7 \leqq \log k \leqslant-2.5$ (cell sizes 1000 to $16000 \mathrm{~km}$ ). In the 10 August plage no wavenumber appeared definitely damped except possibly at the shortest wavelenghts. The results do not reproduce the predictions of one model which represents the photospheric oscillations as gravity waves (Chen and Lykoudis, 1972) although gravity waves propagating in an inclined magnetic field (Michalitsanos, 1973b) are not ruled out.

\section{Acknowledgements}

I am indebted to D. Lynch for discussions of his numerical experiments with artificial power spectra. Thanks are extended to Drs G. A. Paulikas and E. B. Mayfield for an opportunity to study at The Aerospace Corporation, and to J. Frawely for much help with programming. Drs G. A. Chapman and E. N. Frazier were a constant source of stimulation and ideas.

\section{References}

Beckers, J. M. and Parnell, R. L.: 1969, Solar Phys. 9, 39.

Blackman, R. B. and Tukey, J. W.: 1958, The Measurement of Power Spectra, Dover Publications, New York.

Canfield, R. C. and Mehltretter, J. P.: 1973, Solar Phys. 33, 33. 
Chen, C.-J. and Lykoudis, P. S.: 1972, Solar Phys. 25, 380.

Deubner, F. L.: 1969, Solar Phys. 9, 343.

Frazier, E. N.: 1971, in R. Howard (ed.), 'Solar Magnetic Fields', IAU Symp. 43, 260.

Giovanelli, R. G. and Ramsay, J. V.: 1971, in R. Howard (ed.), 'Solar Magnetic Fields', IAU Symp. 43, 293.

Gonczi, G. and Roddier, F.: 1969, Solar Phys. 8, 255.

Howard, R.: 1967, Solar Phys. 2, 3.

Howard, R., Tannenbaum A. S., and Wilcox, J. M.: 1968, Solar Phys. 4, 286.

Leighton, R. B., Noyes, R. W., and Simon, G. W.: 1962, Astrophys. J. 135, 474.

Leibacher, J. and Stein, R. F.: 1971, Astrophys. Letters 7, 191.

Lynch, D.: 1974, private communication.

Mattig, W., Mehltretter, J. P., and Nesis, A.: 1969, Solar Phys. 10, 254.

Mayfield, E. B.: 1971, in R. Howard (ed.), 'Solar Magnetic Fields', IAU Symp. 43, 376.

McKenzie, J. F.: 1971, Astron. Astrophys. 15, 450.

Michalitsanos, A. G.: 1973a, Earth Extraterrest. Sci. 2, 125.

Michalitsanos, A. G.: 1973b, Solar Phys. 30. 73.

Nakagawa, Y., Priest, E. R., and Wellck, R. E.: 1973, Astrophys. J. 184, 931.

Reiling, H.: 1971, Solar Phy's. 19, 297.

Sheeley, Jr., N. R. and Engvold, O.: 1970, Solar Phys. 12, 69.

Stenflo, J. O.: 1973, Solar Phys. 32, 41.

Stix, M.: 1970, Astron. Astrophys. 4, 189.

Teske, R. G.: 1974a, Solar Physics, in press.

Teske, R. G.: 1974b, Aerospace Technical Report, ATR-75 (8102)-1

Thomas, J. H., Clark, P. A., and Clark, Jr., A.: 1971, Solar Phys. 16, 51.

Uchida, Y.: 1965, Astrophys. J. 142, 335.

Ulrich, R. K.: 1970, Astrophys. J. 162, 993.

White, O. R. and Cha, M. Y.: 1973, Solar Phys. 31, 23. 